\title{
Blood and Intestinal Parasites of Squirrels (Rodentia: Sciuridae) in Amazonian Brazil
}

\section{Ralph Lainson $/{ }^{+}$, Maria do Carmo de 0 liveira Brígido*, Fernando Tobias Silveira}

\author{
Departamento de Parasitologia, Instituto Evandro Chagas, Av. Almirante Barroso 492, 66090-000 Belém, PA, Brasil \\ *Instituto Brasileiro do Meio Ambiente e de Recursos Naturais Renováveis, Belém, PA, Brasil
}

We report the result of an examination for blood and intestinal protozoa in 12 specimens of the red squirrel Sciurus spadiceus (Rodentia: Sciuridae) from Birroque, municipality of Plácido de Castro, state of Acre, Brazil. No parasites were detected in thin, Giemsa-stained blood films of the animals, but culture of the blood of three in Difco B45 medium blood-agar slants gave rise to isolates of epimastigotes. Inoculation of one isolate into laboratory mice resulted in the appearance of Trypanosoma cruzi-like trypomastigotes in their peripheral blood, and the other two isolates gave rise to transient infections with a $\mathrm{T}$. lewisi-like parasite in inoculated mice and hamsters. The failure of the latter parasite to develop in the triatomine bug Rhodnius robustus suggests that it is probably not $\mathrm{T}$. rangeli. This appears to be the first record of a $\mathrm{T}$. lewisi-like trypanosome in neotropical squirrels. Oocysts of an Eimeria sp., were detected in the faeces of 10 animals (83.3\%). The parasite develops in the epithelial cells of the intestine, where it may cause severe damage and sometimes results in death of the animal. No oocysts were detected in bile.

Key words: Sciurus spadiceus - squirrels - trypanosomes - Eimeria - Brazil

During 2001-2002, a parasitological study was made of 12 specimens of the red squirrel Sciurus spadiceus cap-

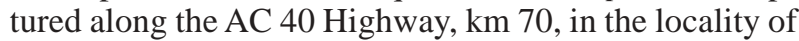
Birroque, municipality of Plácido de Castro, state of Acre, North Brazil. Blood was obtained from the femoral vein of the animals for preparation of thin blood films and culture in Difco B45 medium blood-agar slants. Air-dried blood films were fixed in absolute methyl alcohol and stained by Giemsa's method. Cultures were checked at weekly intervals and only discarded if negative at one month.

Faecal samples from the squirrels were lightly triturated in $2 \%$ aqueous potassium dichromate solution $\left(\mathrm{K}_{2} \mathrm{Cr}_{2} \mathrm{O}_{7}\right)$ and spread as a thin layer in covered Petri dishes kept at approximately $24-26^{\circ} \mathrm{C}$. They were examined at once for the presence of coccidial oocysts, using normal light microscopy at magnifications of $\mathrm{x} 160$ and $\mathrm{x}$ 400 and then at daily intervals to follow development of any oocysts detected. Bile was removed from the gall bladder of autopsied animals and also examined for oocysts. Tissues for histology were fixed in $10 \%$ buffered formol-saline, embedded in paraffin wax and examined in sections cut at $4 \mu \mathrm{m}$ and stained with haematoxylin and eosin. Photomicrographs were made with a Zeiss "Photomicroscope III" and Kodak TMX 100 film.

Financial support: Ministry of Health, Secretaria de Vigilância em Saúde, Brazil and grant 066445 from the Wellcome Trust, London (FTS and RL)

${ }^{+}$Corresponding author. Fax: +55-91-226.1284. E-mail: ralphlainson@iec.pa.gov.br

Received 15 April 2004

Accepted 3 September 2004
No parasites were detected in the Giemsa-stained blood films of the squirrels, but growth of epimastigotes occurred in the cultures of blood from three of them. The culture forms of one isolate were morphologically quite different from those of the other two cultures, and when inoculated into laboratory mice produced trypomastigotes which were morphologically indistinguishable from $T$. cruzi (Fig. 1). The epimastigotes of the other two cultures were remarkably elongated (Fig. 2) and inoculation of parasites from 10 day-old cultures into mice and hamsters gave rise to infections with a Trypanosoma lewisi-like parasite (Fig. 3). These infections were of a fleeting nature, however, and by the 4th day post-inoculation the trypanosomes had disappeared from the peripheral blood: subsequent attempts to isolate the parasite from the mice following the culture of blood failed.

Initially, morphology of the trypomastigotes led us to suspect that the $T$. lewisi-like parasite might be $T$. rangeli and so, on three occasions, batches of approximately 20 nymphs of the triatomine bug Rhodnius robustus were fed on mice which were showing trypomastigotes in their peripheral blood at two days p.i. Dissection of the bugs made at 1,2, 4, and 6 weeks after the blood-meal showed no development of the parasite in the intestine or the haemolymph.

The type host of $T$. rangeli Tejera, 1920 is the triatomine $R$. prolixus and natural and experimental infections have also been recorded in a wide variety of other triatomine species (D'Alessandro 1976). R. robustus is very closely related to $R$. prolixus (Lent \& Wygodzinsky 1979), to the extent that doubts have been expressed that they represent two distinct species. Our failure to infect $R$. robustus with the T. lewisi-like parasite, together with the fact that this insect has been shown to be a suitable vector of $T$. 
rangeli (D'Alessandro-Bacigalupo \& Gore-Saravia 1999), therefore leads us to suspect that we are dealing with another trypanosome. However, although $R$. prolixus is known to be susceptible to the majority of $T$. rangeli strains, reports do exist of the occasional failure to infect this insect with other isolates (Steindel \& Guarneri 1996) and this clearly indicates our need to investigate the susceptibility of other species of triatomines.

Our attempts to obtain development of this parasite in the mosquitoes Culex quinquefasciatus and Aedes aegypti, and the phlebotomine sand fly Lutzomyia longipalpis were equally unsuccessful. As fleas are vectors of T. lewisi of rats, and highly suspected to transmit species of T. lewisi-like typanosomes of squirrels in the Old World (Hoare 1972), they may well be vectors of the trypanosome in $S$. spadiceus.

In his treatise on the trypanosomes of mammals, Hoare (1972) listed the known species within the subgenus

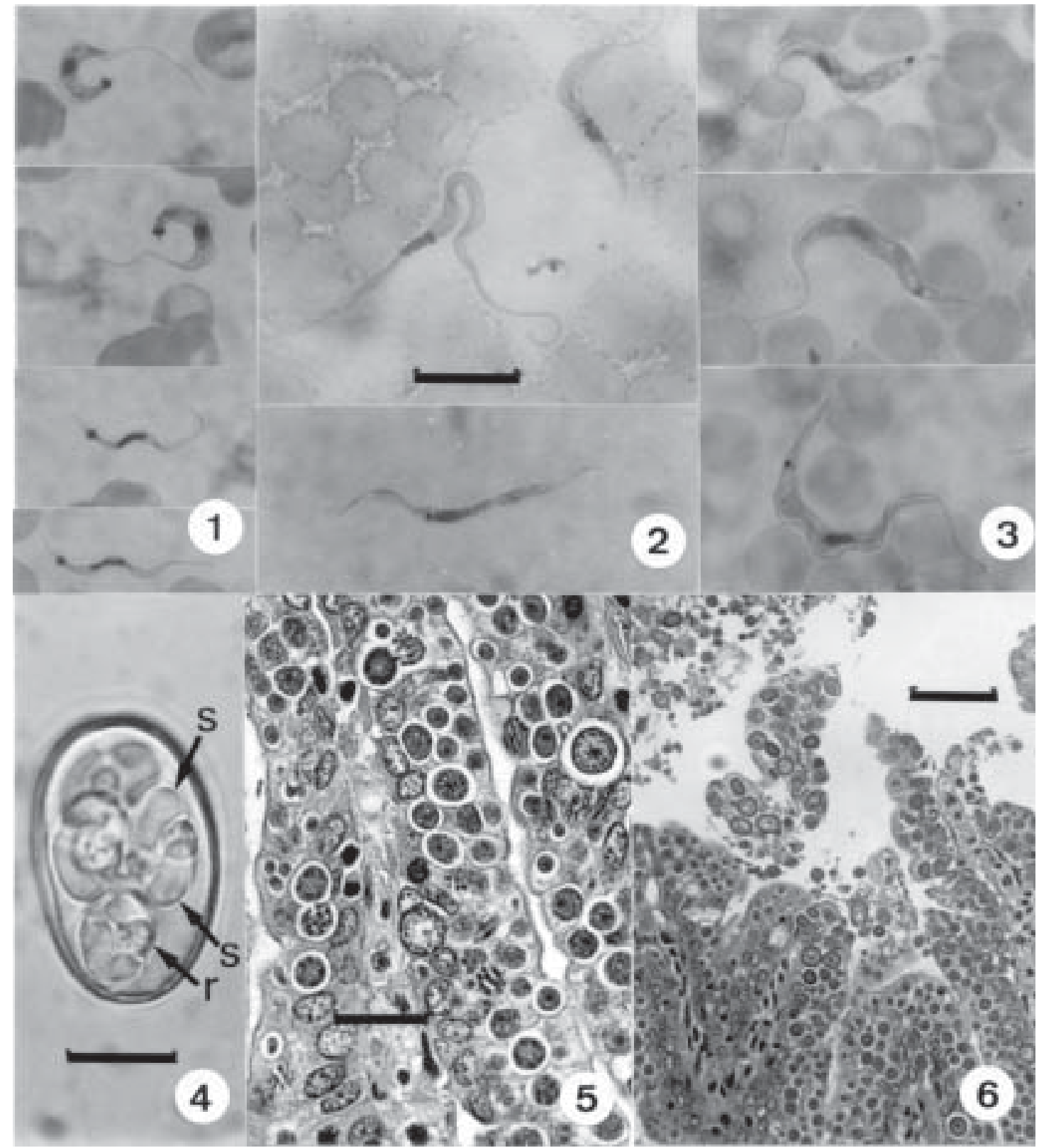

Protozoal parasites of squirrels in Amazonian Brazil. Fig. 1: broad and slender forms of a Trypanosoma cruzi-like trypanosome in the blood of a laboratory mouse inoculated with culture forms of the parasite isolated from the squirrel Sciurus spadiceus. Fig. 2: epimastigotes of a T. lewisi-like trypanosome in a culture of the parasite from S. spadiceus. Fig. 3: trypomastigotes of the T. lewisi-like parasite in the peripheral blood of an experimentally infected laboratory mouse. Fig. 4: mature oocyst of an Eimeria sp. commonly infecting S. spadiceus. S: sporozoite; r: sporocystic residuum. Fig. 5: large numbers of developing meronts and gamonts of the coccidian in a section of the ileum. Fig. 6: low power view of the same material showing vast numbers of parasites and destruction of the intestinal epithelium. Haematoxylin and eosin staining. Bar in Fig. $2=10 \mu \mathrm{m}$ and also applies to Figs 1 and 3. Bar in Fig $4=10 \mu \mathrm{m}$, in Fig. $5=20 \mu \mathrm{m}$ and in Fig. $6=50 \mu \mathrm{m}$ 
Herpetosoma and drew attention to the fact that although T. lewisi-like trypanosomes had been described in North American sciurids (Dorney 1967), there was no record of a similar parasite in neotropical squirrels. We, too, have been unable to trace any such report.

Oocysts of an Eimeria sp., all with similar morphology (Fig. 4 ) were detected in the faeces of 10 (83.3\%) of the squirrels examined for coccidial parasites. A few fully sporulated oocysts were seen, at autopsy, in stained smears of the ileum of a heavily infected animal but sporulation of the majority was only completed at some time within 24 h outside the host; no oocysts were found in the bile of two infected animals that were autopsied.

Endogenous stages developed in the epithelial cells of the small intestine, with massive infections resulting in extensive desquamation of the gut epithelium (Figs 5, 6) and sometimes in death of the animal. Such infections may have been exacerbated by the stress of captivity. This coccidian of Brazilian squirrels is of interest in confirming the severity of coccidiosis in the genus Sciurus, previously noted in the squirrel $S$. aureogaster hypopyrrhus from Belize, Central America (Lainson 1968) and as discussed by Pellérdy (1974).

The taxonomic status of both the T. lewisi-like and $T$. cruzi-like trypanosomes is at present speculative, but it is hoped that continuing studies will provide information on the reproductive phase of their life-cycles and biochemistry which will indicate the parasites' affinities and permit their full descriptions. Depository numbers of two specimens of S. spadiceus are 33535 and 33536.

The present study was carried out within the project "Coccidia and trypanosomatids of the Amazonian fauna of Brazil: taxonomy and life-cycles" approved by the Ethics Committee of the Instituto Evandro Chagas. The subproject "Protozoal parasites of squirrels (Sciurus spp.)" has been approved by the Brazilian Institute for the Environment and Natural Resources (Ibama), Belém.

\section{ACKNOWLEDGEMENTS}

To Dr Sebastião A da Silva Valente for provision of laboratory-bred Rhodnius robustus and Aprigio N de Lima and Antonio $\mathrm{J}$ de Oliveira Monteiro for the maintenance and experimental use of these triatomines. To Domingos M da Souza for invaluable work in the field, and Constância M Franco, Roseli R Braga, Yara LL Jennings, Manoel C de Souza, Iorlando R Barata, Raimundo N Barbosa Pires, and Max Moreira Alves for technical assistance. Dr José de Sousa e Silva Jr., Department of Zoology, Museu Paraense Emilio Goeldi, Belém kindly identified the squirrels.

\section{REFERENCES}

D'Alessandro A 1976. Biology of Trypanosoma (Herpetosoma) rangeli Tejera, 1920. In WHR Lumsden \& DA Evans, Biology of the Kinetoplastida, Vol. I, Academic Press, London, New York, San Fransisco, p. 327-403.

D'Alessandro-Bacigalupo A, Gore-Saravia N 1999. Trypanosoma rangeli. In HM Gilles, Protozoal Diseases, Oxford University Press, Oxford, p. 398-412.

Dorney RS 1967. Incidence, taxonomic relationships and development of lewisi-like trypanosomes in Wisconsin Sciuridae. J Protozool 14: 425-428.

Hoare CA 1972. The Trypanosomes of Mammals, Blackwell Scientific Publication, Oxford, 749 pp.

Lainson R 1968. Parasitological studies in British Honduras III. - Some coccidial parasites of mammals. Ann Trop Med Parasitol 62: 252-259.

Lent H, Wygodzinsky P 1979. Revision of the Triatominae (Hemiptera, Reduviidae), and their significance as vectors of Chagas disease. Bull Amer Museum Natural History, Vol. 163, Article 3, New York.

Pellérdy LP 1974. Coccidia and Coccidiosis, Verlag Paul Parey, Berlin \& Hamburg, 959 pp.

Steindel M, Guarneri A A 1996. Interaction of trypanosomes with different triatomine species. Mem Inst Oswaldo Cruz 91 (Suppl.): 32-33.

Tejera E 1920. Un noveau flagellé de Rhodnius prolixus, Trypanosoma (ou Crithidia) rangeli n.sp. Bull Soc Path Exot 13: 527-530. 
